\title{
Solubilidade e produto de solubilidade
}

Sendo o acompanhamento de reacçōes químicas em solução aquosa, situação corrente, é importante saber "a priori" da facilidade de solubilização do maior número possível de solutos. Daí que a solubilidade seja uma das grandezas características, mencionadas no Handbook of Chemistry and Physics. Informação sobre valores de solubilidade pode obter-se também da consulta de tabelas de produto de solubilidade que figuram em inúmeros livros de texto, particularmente de Química Analítica.

Sendo embora grandezas de uso frequente, solubilidade e produto de solubilidade, nem sempre (1), ao serem mencionadas, ou mesmo utilizadas, aparecem definidas de forma correcta. Tanto uma como outro, para o par soluto-solvente a determinada temperatura, são definidos em situação de equilíbrio dinâmico entre as duas fases - solução saturada e precipitado - logo exigindo a presença simultânea de ambas.

\section{Definição $I$ - SOLUBILIDADE - concentração em solução saturada}

Solubilidade NÃO É o máximo valor da concentração; podem existir soluçð̃es sobre-saturadas.

Definição II - PRODUTO DE SOLUBILIDADE, $\mathrm{K}_{\mathrm{sp}}^{\circ}$ - constante termodinâmica de equilibrio da reacção iónica heterogénea

$\mathrm{M}_{\mathrm{n}} \mathrm{A}_{\mathrm{m}}(\mathrm{s})=\mathrm{nM}^{\mathrm{Z}+}$ (aq.) $+\mathrm{mA} \mathrm{A}^{\mathrm{z}-\text { (aq.) }}$

$\mathrm{K}_{\mathrm{sp}}^{o}=\mathrm{a}_{\mathrm{MZ}+}^{\mathrm{n}} \cdot \mathrm{a}_{\mathrm{AZ}-}^{\mathrm{m}}=\gamma_{ \pm}^{\mathrm{n}+\mathrm{m}} \cdot \mathrm{c}_{\mathrm{M} \mathrm{Z}+}^{\mathrm{n}} \cdot \mathrm{c}_{\mathrm{AZ}-}^{\mathrm{m}}$

onde $\gamma_{ \pm}$representa o coeficiente de actividade médio das espécies, catiónica $\mathrm{M}^{\mathrm{Z}+}$ e aniónica $\mathrm{A}^{\mathrm{Z}-}$ presentes nas concentraçðes $c_{m Z}+e c_{A z}$ e com as actividades $\mathrm{a}_{\mathrm{MZ}+} \mathrm{e} \mathrm{a}_{\mathrm{AZ}-}$, resultantes da dissolução, com dissociação, do soluto $M_{n} A_{m}$.

Como valor aproximado desta constante termodinâmica, é muitas vezes utilizada a grandeza $\mathrm{K}_{\mathrm{sp}}$, experimentalmente acessível

$\mathrm{K}_{\mathrm{sp}}=\mathrm{c}_{\mathrm{M} \mathrm{Z}+}^{\mathrm{n}} \cdot \mathrm{c}_{\mathrm{A} \mathrm{Z}-}^{\mathrm{m}}$

A aproximação

$\mathrm{K}_{\mathrm{sp}}^{\circ} \simeq \mathrm{K}_{\mathrm{sp}}$

é geralmente válida, dado que as soluçð̋es de sais fracamente solúveis, sendo diluídas, podem constituir razoável aproximação das soluçðes ideais, i.e., $\gamma_{ \pm}=1$. No entanto, Produto de Solubilidade NẪO É necessariamente

"O produto das concentraçðes das espécies iónicas em solução”' (1)

ou sequer

"O produto das concentraçð̃es das espécies iónicas em solução, elevadas a um expoente igual ao respectivo coeficiente estequiométrico", como erradamente é vulgar encontrar.

A utilização indevida das designações Solubilidade e Produto de Solubilidade para estas e outras grandezas de utilidade prática discutivel, que não as acima apresentadas, I e II, só pode conduzir a confusðes desnecessárias e a dúvidas, como a que, a título de exemplo, mais adiante se passa a esclarecer.

Solubilidade e Produto de Solubilidade são grandezas relacionáveis em qualquer circunstância, isto é, quer na ausência, quer na presença de iōes comuns e/ou outras espécies para além das que resultam do contacto-precipitado/solvente. No entanto, as relaçðes a seguir apresentadas só são válidas no caso de soluçðes puras do soluțo no solvente, em que as espécies iónicas apenas estão presentes na proporção permitida pela estequiometria do composto, isto é, na ausência de ið̃es comuns ou de reacçð̃es secundárias em que as espécies $\mathrm{M}^{\mathrm{Z}+}$ ou $\mathrm{A}^{\mathrm{z}-}$ estejam envolvidas.

Exemplo 1) - Solução saturada de sal do tipo MA, de solubilidade $\mathrm{s}$

$$
\begin{aligned}
& \mathrm{MA}(\mathrm{s} .)=\underset{\begin{array}{c}
\mathrm{M}^{+} \text {(aq.) } \\
\text { conc. equil. }
\end{array}}{\mathrm{s}}+\underset{\mathrm{s}}{\mathrm{A}^{-} \text {(aq.) }} \begin{array}{c}
\text { conc. equil. } \\
\mathrm{K}_{\mathrm{sp}}^{\mathrm{o}}=\mathrm{a}_{\mathrm{M}+} \cdot \mathrm{a}_{\mathrm{A}-}=\gamma_{ \pm}^{2} \cdot \mathrm{s}^{2} \\
\mathrm{~K}_{\mathrm{sp}}^{\mathrm{o}} \simeq \mathrm{s}^{2}=\mathrm{K}_{\mathrm{sp}} \\
\mathrm{s}=\left(\mathrm{K}_{\mathrm{sp}}\right)^{1 / 2}
\end{array}
\end{aligned}
$$

Exemplo 2) - a) Solução saturada de sal $\mathrm{MA}_{2}$, de solubilidade $\mathrm{s}$

$$
\begin{aligned}
& \mathrm{MA}_{2}(\mathrm{~s} .)=\underset{\mathrm{s}}{\mathrm{M}^{2+}(\alpha \omega .)}+\underset{\text { conc. equil. }}{2 \mathrm{~A}(\mathrm{aq} .)} \\
& \begin{array}{c}
\text { conc. equil } \\
2 \mathrm{~s}
\end{array} \\
& \mathrm{~K}_{\mathrm{sp}}^{\mathrm{o}}=\mathrm{a}_{\mathrm{M}^{2+}} \cdot \mathrm{a}_{\mathrm{A}-}^{2}=\gamma_{ \pm}^{3} \cdot \mathrm{s} \cdot(2 \mathrm{~s})^{2} \\
& \mathrm{~K}_{\mathrm{sp}}^{\mathrm{o}} \simeq 4 \mathrm{~s}^{3}=\mathrm{K}_{\mathrm{sp}} \\
& \mathrm{s}=\left(\frac{K}{4}\right)^{1 / 3}
\end{aligned}
$$

b) Solução saturada de sal MAB, de solubilidade $\mathrm{s}$

$$
\begin{aligned}
& M A B \text { (s.) } \rightleftharpoons \mathrm{M}^{2+} \text { (aq.) }+\mathrm{A}^{-} \text {(aq.) }+\mathrm{B}^{-} \text {(aq.) } \\
& \text { conc. equil. conc. equil. conc. equil. } \\
& S \\
& \mathrm{~S} \\
& \mathrm{~K}_{\mathrm{sp}}^{\circ}=\mathrm{a}_{\mathrm{M}^{2}+} \cdot \mathrm{a}_{\mathrm{A}-} \cdot \mathrm{a}_{\mathrm{B}-}=\gamma_{ \pm}^{3} \cdot \mathrm{s}^{3} \\
& \mathrm{~K}_{\mathrm{sp}}^{\mathrm{o}} \simeq \mathrm{s}^{3}=\mathrm{K}_{\mathrm{sp}} \\
& =\left(K_{s p}\right) 1 / 3
\end{aligned}
$$

a CECUL - Faculdade de Ciências. R. Escola Politécnica 58 1294 Lisboa Codex - Portugal. 
As relaçðes apresentadas, válidas apenas nas condiçðes específicas são por demais conhecidas, aceites e utilizadas.

Por raciocínio análogo poderia prosseguir-se e encontrar relaçðes para outros sais com diferente estequiometria. Chegar-se-ia à relação geral

$S=\left(\frac{K_{s p}}{n^{n} \cdot m^{m} \cdot q^{q}}\right)^{1 / n+m+q}$

para o equilibrio envolvendo o sal de tipo genérico $\mathrm{M}_{\mathrm{n}} \mathrm{A}_{\mathrm{m}} \mathrm{B}_{\mathrm{q}}$ de solubilidade $\mathrm{s}$

$\mathrm{M}_{\mathrm{n}} \mathrm{A}_{\mathrm{m}} \mathrm{B}_{\mathrm{q}}\left(\mathrm{s}\right.$.) $\rightleftharpoons \mathrm{n} \mathrm{M}^{\mathrm{Z}+}$ (aq.) $+\mathrm{mA}^{\mathrm{Z}-}$ (aq.) +

$+\mathrm{q} \mathrm{B}^{\mathrm{Z}-}$ (aq.)

À dúvida que a alguns tem surgido (2), por tentarem, sem resultado, ver no sal $\mathrm{MA}_{2}$ um caso particular de $\mathrm{MAB}$ em que $\mathrm{A}=\mathrm{B}$, vai-se tentar responder de forma inequívoca.

Eventuais esforços em querer ver no sal $\mathrm{MA}_{2}$, onde $s=\left(\frac{K_{s D}}{4}\right)^{1 / 3}$

um caso particular de MAB, onde

$\mathrm{S}=\left(\mathrm{K}_{\mathrm{sp}}\right)^{1 / 3}$

são de facto infrutíferas.

De facto sendo os equilibrios dinâmicos, a probabilidade de $\mathrm{A}^{-}$e $\mathrm{B}^{-}$se encontrarem em solução de $\mathrm{M}^{+}$, $\mathrm{A}^{-}$e $\mathrm{B}^{-}$, para formarem $\mathrm{MAB}$ é inferior à probabilidade de partículoas $\mathrm{A}^{-}$se encontrarem em solução de $\mathrm{M}^{+}$e $\mathrm{A}^{-}$para formarem $\mathrm{MA}_{2}$ (3).

Tal pode ser provado invocando relaçðes termodinâmicas do verdadeiro conceito de produto de solubilidade $\mathrm{K}_{\mathrm{sp}}^{\circ}$, que ao contrário de $\mathrm{s}$ e de $\mathrm{K}_{\mathrm{sp}}$, não varia pela adição de outros sais (4), e que permite o cálculo da -variação padrão da energia de Gibbs para a reacção de solubilização

$\Delta_{\text {sol. }} \mathrm{G}^{\circ}=-\mathrm{RT} \ln \mathrm{K}_{\mathrm{sp}}^{\circ}$

Eventuais diferenças

$\delta \Delta_{\text {sol. }} \mathrm{G}^{\mathrm{o}}=\Delta_{\text {sol. }} \mathrm{G}_{\mathrm{MAB}}^{\mathrm{o}}-\Delta_{\text {sol. }} \mathrm{G}_{\mathrm{MA}_{2}}^{\mathrm{o}}$

são justificadas em termos de contribuiçð̃es entrópicas, uma vez que

$\delta \Delta_{\text {sol. }} \mathrm{G}^{\circ}=\delta \Delta_{\text {sol. }} \mathrm{H}^{\circ}-\mathrm{T} \delta \Delta_{\text {sol. }} \mathrm{S}^{\mathrm{o}}$

e para soluçðes ideiais é $\delta \Delta_{\text {sol. }} \mathrm{H}^{\circ}=0$

ou seja, o princípio entrópico da individualidade das partículas conduz a que

$\Delta_{\text {sol. }} \mathrm{G}_{\mathrm{MA}_{2}}^{\mathrm{o}}=-\mathrm{RT} \ln \mathrm{K}_{\mathrm{sp}\left(\mathrm{MA}_{2}\right)}^{\mathrm{o}} \simeq-\mathrm{RT} \operatorname{ln~} \mathrm{K}_{\mathrm{sp}\left(\mathrm{MA}_{3}\right)}=$ $=-\mathrm{RT} \ln 4 \mathrm{~s}^{3}$

$\Delta_{\text {sol. }} \mathrm{G}_{\mathrm{MAB}}^{\mathrm{o}}=-\mathrm{RT} \ln \mathrm{K}_{\mathrm{sp}(\mathrm{MAB})}^{\mathrm{o}} \simeq-\mathrm{RT} \ln \mathrm{K}_{\mathrm{sp}(\mathrm{MAB})}=$ $=-\mathrm{RT} \ln \mathrm{s}^{3}$

$\delta \Delta_{\text {sol. }} \mathrm{G}^{\circ}=\Delta_{\text {sol. }} \mathrm{G}_{\mathrm{MAB}}^{\mathrm{o}}-\Delta_{\text {sol. }} \mathrm{G}_{\mathrm{MA}_{2}}^{\mathrm{o}} \simeq-\mathrm{RT} 1 \mathrm{n} \mathrm{m}^{3}+$ $+\mathrm{RT} \ln 4 \mathrm{~m}^{3}=\mathrm{RT} \ln 4$

que a $25^{\circ} \mathrm{C}$ se traduz em

$\delta \Delta_{\text {sol. }} \mathrm{G}^{\circ}=3,436 \mathrm{KJ} \cdot \mathrm{mol}^{-1}$.

\section{REFERÊNCIAS}

(1) Handbook of Chemistry and Physics CRC Press - 56th edition - 1975-1976 - F. 115.

(2) S. Parthasarathy Chemistry in Britain, 21, 27 (1985).

(3) M.R. Masson ibid., 21, 244 (1985).

(4) G. Kortüm Treatise on Electrochemistry Elsevier Sc. Pub. Co., Amsterdam - 1965, pg. 163.

\section{COLABORE COM A SOCIEDADE}

